\title{
Steroid reference intervals in women: influence of menopause, age and metabolism
}

\section{Marco Mezzullo, Alessandra Gambineri, Guido Di Dalmazi ${ }^{\circledR}$, Alessia Fazzini, Matteo Magagnoli, Margherita Baccini, Valentina Vicennati, Carla Pelusi, Uberto Pagotto and Flaminia Fanelli}

Department of Medical and Surgical Sciences, Unit of Endocrinology and Prevention and Care of Diabetes, Center for Applied Biomedical Research, University of Bologna, S.Orsola Policlinic, Bologna, Italy
Correspondence should be addressed to F Fanelli

Email

flaminia.fanelli2@unibo.it

\begin{abstract}
Objective: To investigate the impact of age, obesity and metabolic parameters on 13 circulating steroids in reproductive and menopausal age. To define reference intervals (RIs).

Design: Cross-sectional.

Methods: Three hundred and twenty five drug-free, healthy and eumenorrheic women were selected from the general population. Independent relationships of LC-MS/MS-determined steroid levels with age, BMI and metabolic parameters were estimated. Reference sub-cohorts were defined for calculating upper and lower limits in reproductive age, menstrual phases and menopause, and these were compared with limits in dysmetabolic sub-cohorts. Results: Lower androgens, pro-androgens and estrogens, but higher cortisol and metabolites were found in menopausal compared to reproductive age women. Androgens and precursors decreased during reproductive age $(P<0.001-P=0.002)$ but not after menopause. 170H-progesterone decreased with BMI $(P=0.006)$ and glucocorticoids with waist circumference $(P<0.001-P=0.002)$ in reproductive age, but increased with triglycerides $(P=0.011-P=$ $0.038)$ after menopause. Inverse associations of dihydrotestosterone with BMI $(P=0.004)$ and HDL-cholesterol $(P=$ $0.010)$, estrone with total cholesterol $(P=0.033)$ and estradiol with triglycerides $(P=0.011)$ were found in reproductive age. After menopause, estrone increased with waist circumference $(P<0.001)$ and decreased with insulin resistance $(P=0.012)$. Ovarian steroid RIs were estimated in menstrual phases and menopause. Age- and reproductive statusspecific RIs were generated for androgens, precursors and corticosteroids. Lower limits for reproductive age cortisol $(P=0.020)$ and menopausal 11-deoxycortisol $(P=0.003)$ in dysmetabolic sub-cohorts were reduced and increased, respectively, compared to reference limits.

Conclusions: Obesity and dysmetabolism differently influence circulating steroids in reproductive and menopausal status. Age, menstrual and menopausal status-specific RIs were provided by LC-MS/MS for a broad steroid panel.
\end{abstract}

\section{Introduction}

LC-MS/MS is used by an increasing number of clinical laboratories, providing reliable determinations of steroid hormones for the diagnosis and management of endocrine diseases $(1,2)$. By enlarging the panel of measurable precursors and metabolites, LC-MS/MS is also boosting renewed interest in understanding the steroid system derangement in several conditions. However, translating (c) 2021 European Society of Endocrinology Printed in Great Britain research findings into clinical advancements require the definition of pathophysiologic states influencing the circulating steroid levels, as well as of appropriate reference intervals (RI) allowing the effective interpretation of laboratory results (3).

Recently, LC-MS/MS has been applied to study the steroid profile of adrenal tumors (4), type I diabetes (5),

Published by Bioscientifica Ltd. 
or female hyperandrogenism $(1,6,7,8,9)$. In contrast, only a few studies were purposely designed to define steroid RI. Moreover, some studies relied on healthcareseeking subjects. This represents an intrinsic bias for RI estimation, given the adaptive nature of steroid hormones to stressful or debilitating conditions. Other studies did not report an exhaustive characterization of subjects, for example aimed at detecting subtle hyperandrogenic states manifesting as menstrual irregularities or hirsutism, while others tolerated excess weight (10).

Overweight and obesity affect half of the female population $(11,12)$. Steroids are involved in systemic and intracrine mechanisms that, once dysregulated, contribute to excess fat, mostly of abdominal type, hypertension, impaired glucose control and dyslipidaemia. However, the interplay among glucocorticoids, androgens and estrogens in such mechanisms varies with the menopausal transition $(12,13)$. Androgen excess, ovarian dysfunction and metabolic impairment represent a vicious cycle in young women, however, a variegated spectrum of manifestations is observed, for which effective hormonal markers are yet undefined (14). The androgen decline and the loss of ovarian hormones contribute to the central redistribution of body fat, the increased cardiometabolic risk, sarcopenia and bone frailty experienced after menopause. Besides, the modulation of adrenal function throughout women lifespan, and the interplay between the hypothalamuspituitary-adrenal (HPA) axis dysregulation and obesity before and after menopause were not elucidated $(15,16)$.

In a recent study, we demonstrated that the circulating steroid profile in men is not only influenced by aging, but also by obesity and metabolic derangement, and we provided age-specific RI estimated in appropriate reference cohorts (17). In the present study, by using a similarly standardized procedural approach, we aimed at describing the relationships of age, obesity and metabolic status with 13 circulating steroids in women in reproductive and menopausal age. Based on the depicted associations, we generated age, menopause and menstrual phase specific RI in selected reference sub-cohorts, and investigated whether unrestricted inclusion criteria could bias steroid RI estimation.

\section{Subjects and methods}

\section{Subjects}

Women aged 18-86 years were recruited from the general population (18). The study was approved by the S.Orsola
Policlinic ethical committee $(85 / 2008 / \mathrm{O} /$ Tess $)$. All women signed the informed consent before they were interviewed and examined by a trained endocrinologist, between 08:00 $\mathrm{h}$ and 10:00 $\mathrm{h}$. Waist circumference and BMI were recorded. systolic (SBP) and diastolic (DBP) blood pressures were measured in supine position after 3 min rest. Inclusion criteria were: $B M I \geq 18.5 \mathrm{~kg} / \mathrm{m}^{2}$, weight stability in previous 3 months and complete sexual development. Exclusion criteria included signs of clinical hyperandrogenism, history of menstrual irregularities, steroidal (including estro-progestogen) and non-steroidal drug assumption in previous 3 months (except antipyretic or non-steroidal anti-inflammatory compounds tolerated before the previous month, and thyroxine replacement in compensated hypothyroidism), present or past endocrine, hepatic, renal, oncologic, autoimmune, cardiovascular, hematologic, neurologic or psychiatric diseases, sleep disorders, shift working, frequent flying or allergies requiring treatment. Among 653 women examined, 328 were excluded because of present or previous diseases $(n=32)$, irregular sleeping $(n=80)$, glucose-lowering $(n=3)$, cholesterol-lowering $(n=111)$, anti-hypertensive $(n=171)$ and anti-depression $(n=40)$ drug assumption. Therefore, 325 women were included in the present study.

\section{Biochemical and hormonal evaluation}

Blood was withdrawn in overnight fasting condition in Vacuette $\mathrm{Z}$ serum beads clot activator tubes (Greiner Bio-One, Kremsmunster, Austria) after $10 \mathrm{~min}$ saline infusion for minimizing venepuncture stress. After $20 \mathrm{~min}$ settling, tubes were centrifuged $(2000 \mathrm{~g}, 10 \mathrm{~min}$, room temperature) and serum for LC-MS/MS was stored at $-80^{\circ} \mathrm{C}$. Routine hormones and biochemicals were measured in fresh blood as previously reported (17). The homeostatic model assessment-insulin resistance (HOMA-IR) was computed (19).

\section{Steroid measurement by LC-MS/MS}

We applied two in-house LC-MS/MS assays (Supplementary Table 1, see section on supplementary materials given at the end of this article) including 17-hydroxypregnenolone (17OHP5), dehydroepiadrosterone (DHEA), progesterone (P4), 17-hydroxyprogesterone (17OHP4), 11-deoxycorticosterone (DOC), corticosterone (B), 
11-deoxycortisol (11S), cortisol (F), androstenedione (A4), testosterone $(\mathrm{T})$, dihydrotestosterone (DHT), estrone (E1) and estradiol (E2) $(18,20,21)$.

\section{Study design}

Women were classified in reproductive (regular menses, age $18-54$ years; $n=186$ ) or menopausal (amenorrhea in previous 12 months or more, age $48-86$ years; $n=127$ ) age, and further subdivided in normal weight (NW, $18.5<\mathrm{BMI} \leq 25.0 \mathrm{~kg} / \mathrm{m}^{2} ; n=123$ and 63 , respectively), overweight (OW, $25.0<\mathrm{BMI} \leq 30.0 \mathrm{~kg} / \mathrm{m}^{2} ; n=35$ and 49, respectively) and obese (OB, BMI $>30.0 \mathrm{~kg} / \mathrm{m}^{2} ; n=28$ and 15 , respectively). Women in reproductive age were further stratified in early follicular (day $1-6 ; n=31$ ), preovulatory (day 9-13; $n=30$ ) and mid-luteal (day 18-24; $n=33$ ) menstrual phases. Perimenopausal women were also identified $(<6$ menstrual bleedings in previous 6 months or more, age $48-54$ years; $n=12$ ). Anthropometric, metabolic and steroid values were compared between reproductive and menopausal cohorts, among BMI classes and among menstrual phases. Afterward, we assessed the independent effect of age, BMI, waist circumference, SBP, DBP, HOMA-IR, total cholesterol, HDL-cholesterol and triglycerides on each steroid in reproductive and menopausal ages. According to the results, we defined steroid-specific reference sub-cohorts by excluding women displaying alterations in metabolic parameters influencing the steroid levels. Therefore, we identified subjects with normal ( $\leq 88 \mathrm{~cm}$, norWC; $n=216)$ or elevated $(>88 \mathrm{~cm}$, dysWC; $n=106)$ waist circumference, normal $(<2.5$, norHOMA; $n=194)$ or elevated $(\geq 2.5$, dysHOMA; $n=36)$ HOMA-IR, normal $(<5.17 \mathrm{mmol} / \mathrm{L}$, norTC; $n=194)$ or elevated ( $\geq 5.17 \mathrm{mmol} / \mathrm{L}$, dysTC; $n=123)$ total cholesterol, normal ( $\geq 1.29 \mathrm{mmol} / \mathrm{L}$, norHDL; $n=203)$ or reduced $(<1.29 \mathrm{mmol} / \mathrm{L}$, dysHDL; $n=55)$ HDL-cholesterol, and normal $(<1.69 \mathrm{mmol} / \mathrm{L}$, norTG; $n=302)$ or elevated ( $\geq 1.69 \mathrm{mmol} / \mathrm{L}$, norTG; $n=15)$ triglycerides. Lower (LRL) and upper (URL) reference limits defining the central 95\% of steroid distribution were estimated in reference subcohorts. Age-specific LRL and URL were estimated when required. Finally, to evaluate whether altered metabolic parameters could influence RI estimation, LRL and URL were compared with lower and upper limits calculated in dysmetabolic sub-cohorts including subjects with alterations in the parameters influencing that particular steroid, respectively.

\section{Statistical analysis}

Box-Cox transformation was used for variables showing a significant skewness at the Kolmogorov-Smirnov test (22). Far outliers at the Tukey's test were removed (23). Variables were compared between reproductive age and menopausal cohorts by $t$-test. The ANOVA trend test was used for comparing BMI classes (SPSS package v.20, IBM Co). Comparisons among menstrual phases were performed by one-way ANOVA. The stepwise multiple regression included age, BMI, waist circumference, SBP, DBP, HOMA-IR, total-cholesterol, HDL-cholesterol and triglyceride levels as covariates, and each steroid as dependent variable. The menstrual phase was added as cofactor for steroids varying with the menstrual cycle. The effect size $\left(f^{2}\right)$ was estimated as $f^{2}=\frac{s r^{2}}{\left(1-R^{2} f u l l\right)}$, where $s r^{2}$ is the semipartial correlation coefficient for the predictor of interest, and $\mathrm{R}$ is the full correlation coefficient obtained by the multiple regression model (24).

LRL and URL were estimated as the mean and $+(1.96 \times$ s.D. $)$ of the transformed variables, respectively, then, values were back-transformed to the original unit (25). Age-specific RI was estimated by modeling the transformed steroid variable on age distribution, according to the fractional polynomial regression by Royston and Wright (26). Age $(X)$ was transformed in order to stabilize the steroid variable $(Y)$ for large values of $X$ according to the formula: $e X=\exp \frac{(\log (0.01) \times(X-\min (X)))}{(\max (X)-\min (X))}$. Then, we selected the optimal model providing the lowest polynomial degree (parsimony) with maximum decrease in deviance (goodness of fit). Best-fit polynomial coefficients were selected by fp syntax, and RI was visually inspected by xrigls syntax in STATA (v.13.0, StataCorp LLC).

A large number of cases showed values below the sensitivity limit for DOC in the whole cohort ( $n=276$ of $325)$, and P4, DHT and E2 in menopausal cohort $(n=90$, 75 and 115 of 127, respectively) (Supplementary Table 1). Therefore, DOC comparisons were performed by KruskalWallis and Mann-Whitney tests, while no multiple regression was performed for DOC and for P4, DHT and E2 in menopausal cohort. RI for DOC and P4, and menopausal RI for DHT and E2 were estimated as the 2.5-97.5 centiles of distribution. Lower and upper limits were compared between reference and dysmetabolic sub-cohorts by $\mathrm{z}$ distribution. Two-tailed $P$ values $<0.05$ were considered significant. Data were analyzed by MedCalc Software (v.18.2.1, Mariakerke, Belgium) except where specified. 


\section{Results}

\section{Anthropometric, metabolic and hormonal features of the cohort}

Worsening metabolic parameters at increasing BMI were observed both in reproductive age (min-max BMI: $18.5-42.9 \mathrm{~kg} / \mathrm{m}^{2}$ ) and menopausal women (min-max BMI: $18.9-41.2 \mathrm{~kg} / \mathrm{m}^{2}$ ). In reproductive age, levels of B $(P<0.001), \mathrm{F}(P=0.008)$ and DHT $(P=0.006)$ decreased with increasing BMI classes, with $\mathrm{OB}$ showing lower $\mathrm{B}$, $\mathrm{F}$ and DHT compared to NW $(P=0.002, P=0.023$ and $P=0.017$ ) and lower $\mathrm{B}$ and $\mathrm{F}$ compared to OW (both $P<0.001)$ women. In menopausal women, E1 levels increased with BMI classes $(P=0.002)$, with both OW $(P=0.046)$ and $\mathrm{OB}(P=0.007)$ displaying higher values than NW women (Table 1). Women in different menstrual phases displayed similar anthropometric and metabolic parameters, but different levels of P4, 17OHP4, E1, E2 (all $P<0.001)$, DOC $(P=0.043)$ and $11 \mathrm{~S}(P=0.048)$. In particular, women in mid-luteal phase had higher $\mathrm{P} 4$ and $17 \mathrm{OHP} 4$ as compared to early follicular and pre-ovulatory (all $P<0.001$ ), and higher DOC compared to pre-ovulatory $(P=0.047)$ women. Moreover, lower E1 and E2 levels were found in early follicular compared to pre-ovulatory and mid-luteal phases (all $P<0.001$ ) (Supplementary Table 2).

\section{Independent impact of age, anthropometric and metabolic parameters on steroid levels}

Stepwise multiple regression results are detailed in Table 2 . In women in reproductive age, 17OHP5, DHEA, A4, T (all $P<0.001)$ and DHT $(P=0.002)$ decreased with age, with a large effect size for A4 $\left(f^{2}=0.28\right)$, DHEA and $\mathrm{T}$ (both $\left.f^{2}=0.22\right)$, and moderate for 17OHP5 $\left(f^{2}=0.12\right)$ and DHT $\left(f^{2}=0.11\right)$. DHT was also negatively associated with BMI $\left(P=0.004, f^{2}=0.09\right)$ and HDL-cholesterol $\left(P=0.010, f^{2}=0.08\right) .17 \mathrm{OHP} 4$ inversely associated with BMI $\left(P=0.006, f^{2}=0.22\right)$, while B $\left(P<0.001, f^{2}=0.20\right)$, $\mathrm{F}$ $\left(P<0.001, f^{2}=0.17\right)$ and 11S $\left(P=0.002, f^{2}=0.09\right)$ inversely associated with waist circumference. Finally, E1 and E2 negatively associated with increasing total cholesterol $\left(P=0.033, f^{2}=0.10\right)$ and triglycerides $\left(P=0.011, f^{2}=0.15\right)$, respectively. In menopausal women, no age and BMI influence were detected on steroid levels. 17OHP4 $\left(P=0.026, f^{2}=0.06\right), 11 S\left(P=0.011, f^{2}=0.07\right)$ and $\mathrm{F}$ $\left(P=0.038, f^{2}=0.05\right)$ directly associated with triglycerides. E1 directly associated with waist circumference $(P<0.001$, $\left.f^{2}=0.16\right)$, and inversely associated with HOMA-IR $\left(P=0.012, f^{2}=0.08\right)$.

\section{Steroid reference intervals}

Age- and menopause-specific RI were estimated for 17OHP5, DHEA, A4, T and DHT (Table 3). All women were included in the reference sub-cohort for 17OHP5, DHEA, A4 and T $(n=325)$. For DHT, the reference subcohort included women in reproductive age who were both NW and norHDL, and all menopausal women ( $n=65$ and $n=127$, respectively). Androgens and precursors peaked around age 25 years and progressively declined. A reduction in LRL and URL was found for A4 (-83.2 and $-57.5 \%$, respectively), DHEA (-67.6 and $-56.0 \%$, respectively), 17OHP5 (-49.9 and $-43.1 \%$, respectively) and $\mathrm{T}$ (-24.9 and $-17.2 \%$, respectively), as well as in DHT URL (-56.3\%) from age 25 to 65 years (Fig. 1).

Reproductive age RI were estimated in the whole cohort for DOC $(n=186)$, and in norWC sub-cohort for $\mathrm{B}, 11 \mathrm{~S}$ and $\mathrm{F}(n=132)$. Menopausal RI were estimated in the whole cohort for DOC, B, P4 and E2 $(n=127)$, in norTG sub-cohort for 17OHP4, $11 \mathrm{~S}$ and $\mathrm{F}(n=112)$, and in women who were both norWC and norHOMA for E1 $(n=50)$ (Table 4).

Finally, menstrual phase specific RI were estimated for P4, 17OHP4, E1 and E2 . Of 32 women in mid-luteal phase, 10 (age $39.2 \pm 10.3$ years) exhibited P4 levels $<10 \mathrm{nmol} / \mathrm{L}$ suggestive of incorrect classification or of anovulatory cycle (27), and were therefore excluded. The reference sub-cohorts for early follicular, pre-ovulatory and mid-luteal phase included 31, 30 and 22 women for P4; 22, 26 and 17 NW women for 17OHP4; 20, 25 and 17 norTC women for E1; 25, 28 and 21 norTG women for E2, respectively (Table 5).

\section{Impact of metabolic risk factors on the estimation of reference limits}

LRL and URL of steroids influenced by metabolic parameters were compared with lower and upper limits calculated in dysmetabolic subjects. Higher 11S values were found in the lower limit calculated in dysTG $(n=10)$ compared to LRL in norTG $(n=112)$ menopausal women $(+0.343$ $\mathrm{nmol} / \mathrm{L},+48.4 \%, P=0.003)$. At variance, reduced $\mathrm{F}$ values were found in lower limit in dysWC $(n=52)$ compared with LRL in norWC $(n=132)$ women in reproductive age $(-35.5 \mathrm{nmol} / \mathrm{L},-24.0 \% ; P=0.020$, respectively) (Table 4$)$. 


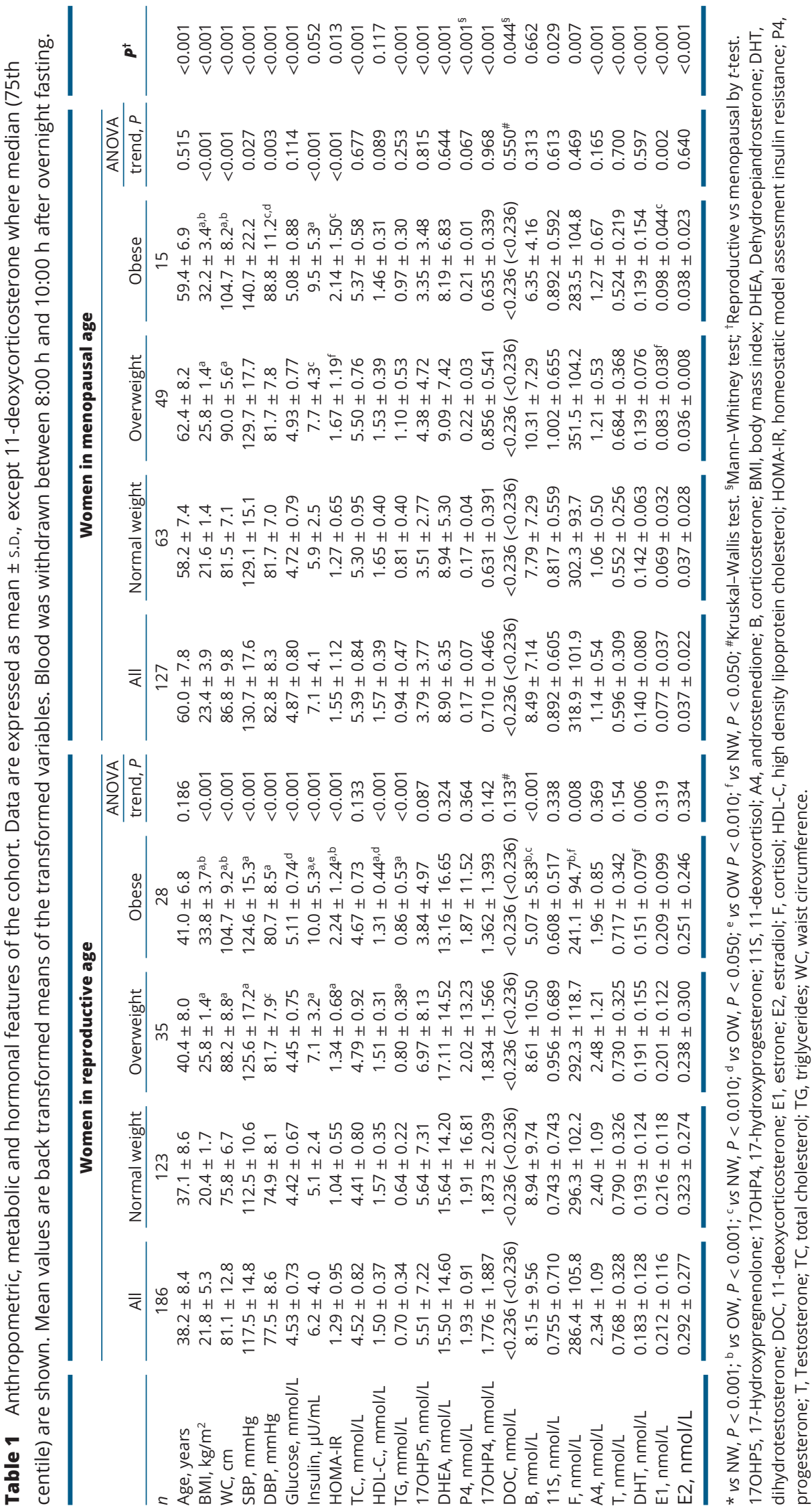




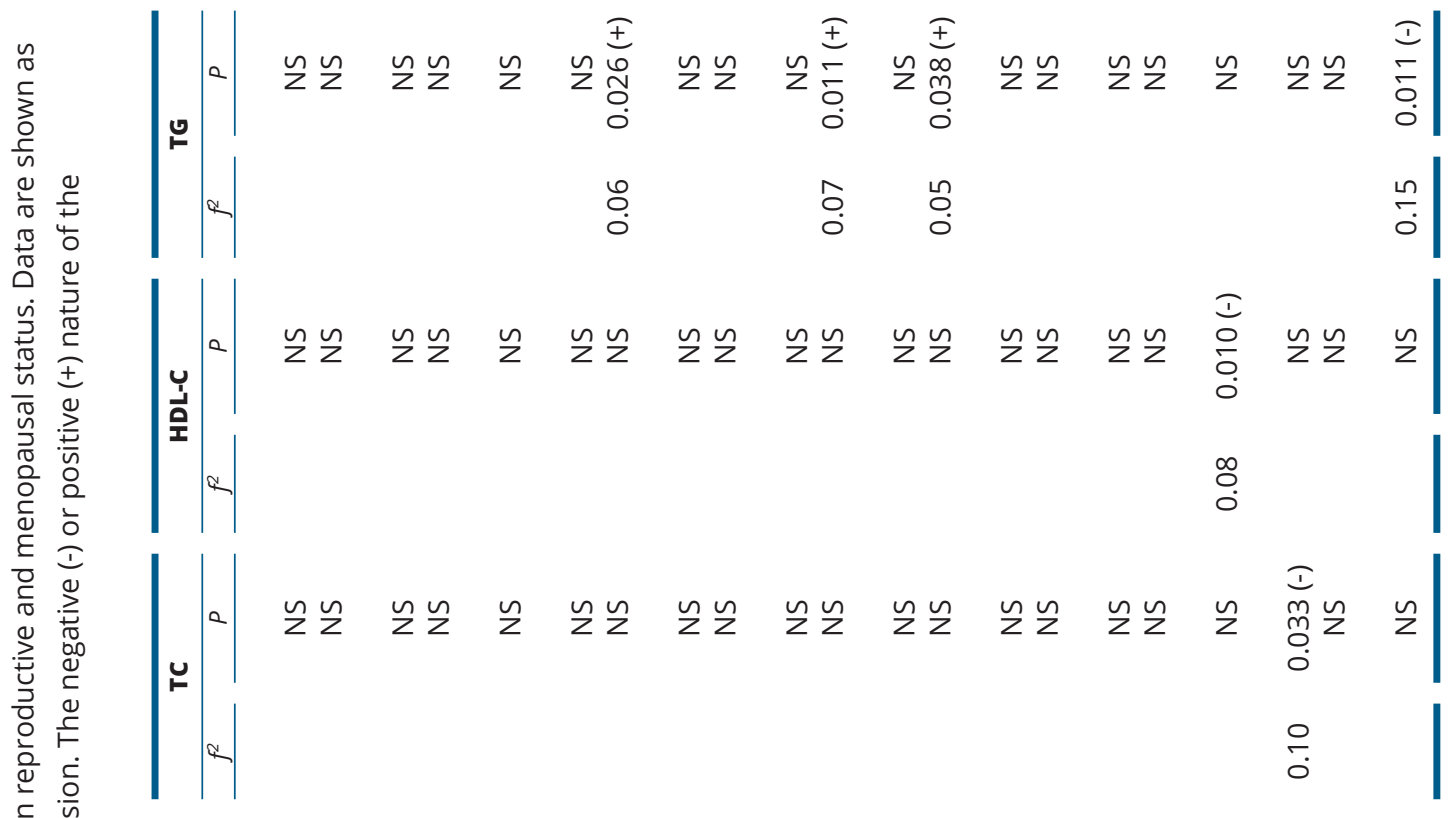

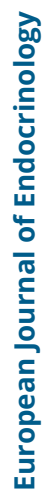

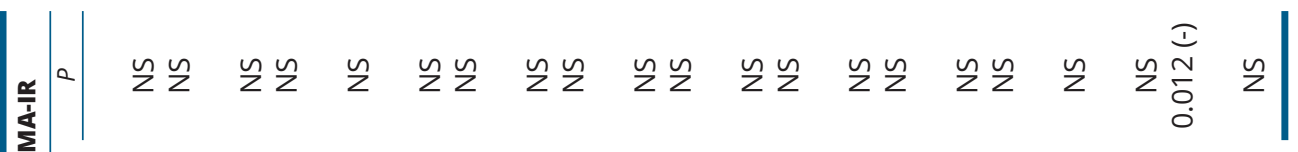
$\stackrel{\infty}{\circ}$

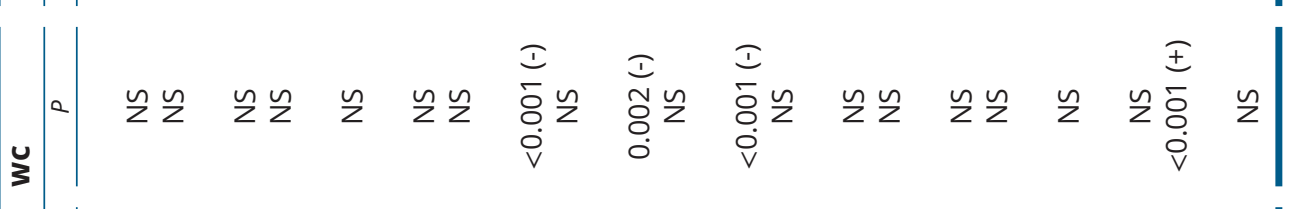
$\stackrel{\text { กิ }}{\circ} \stackrel{\circ}{\circ}$

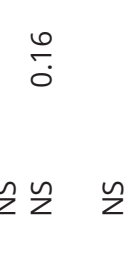
กับ \&े

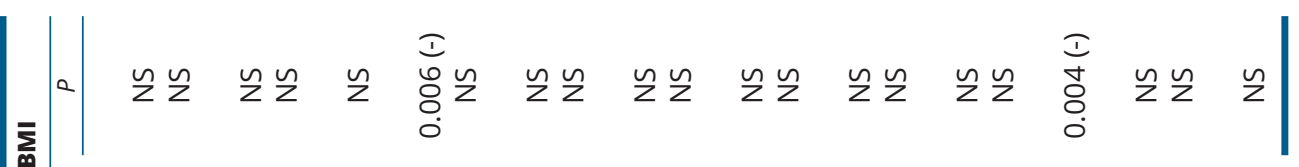
กั่

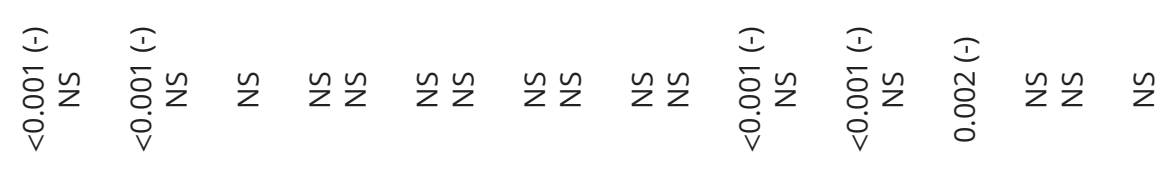
$\stackrel{\sim}{\check{0}} \stackrel{0}{0}$

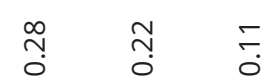




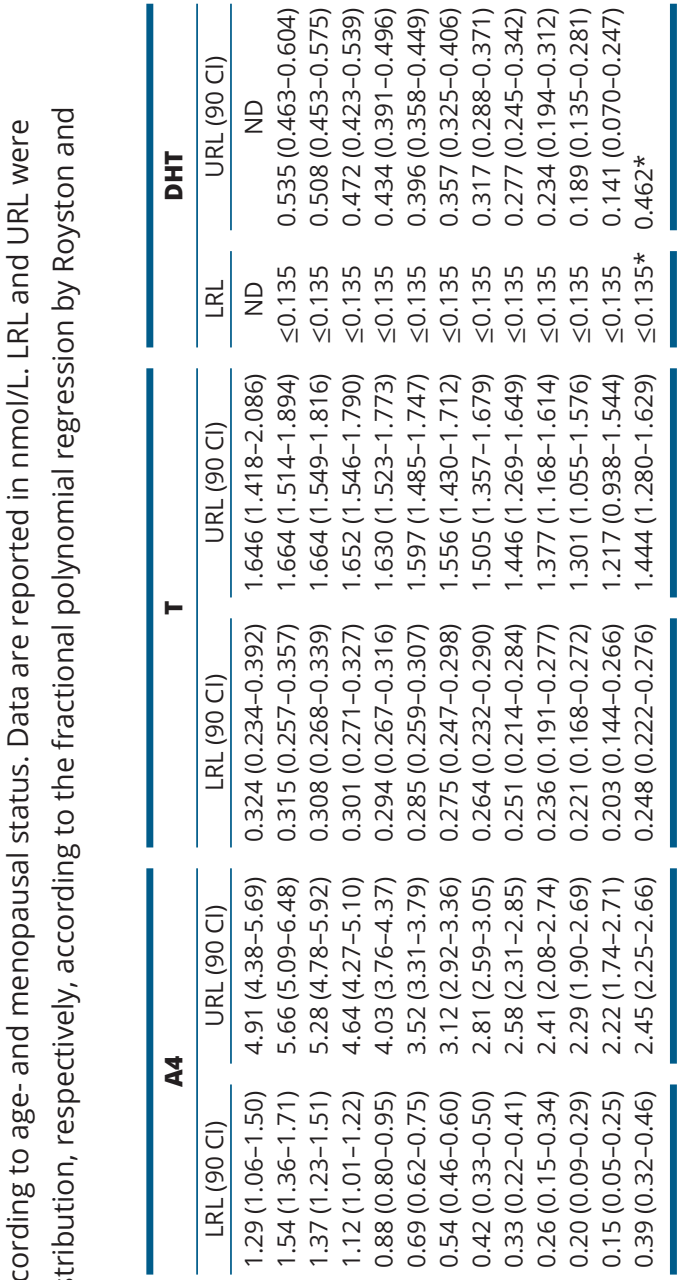

| บิ

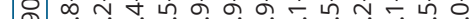

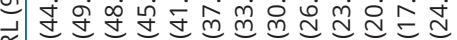

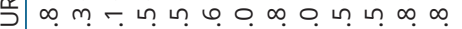
ที่ -

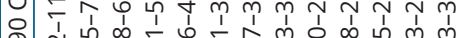
o

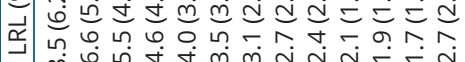
$\mid \begin{array}{ll}\infty \\ 0 \\ 0\end{array}$

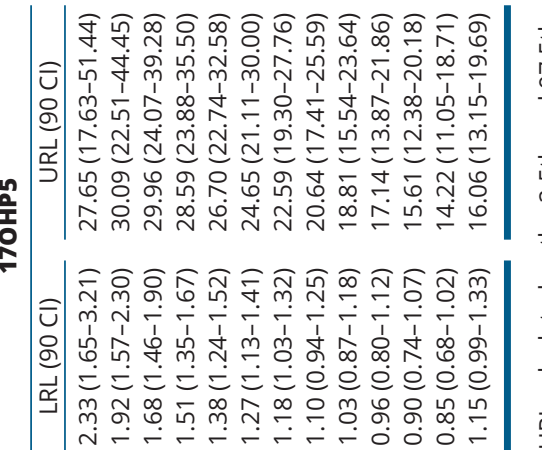
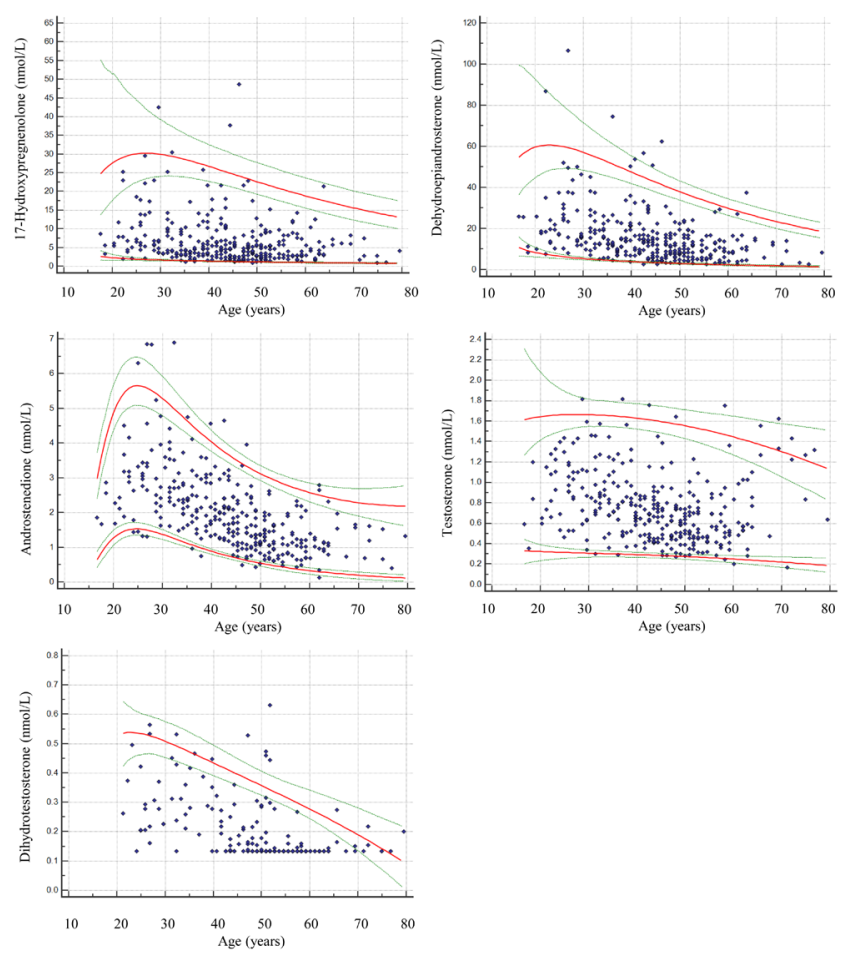

Figure 1

Distribution of steroid hormone serum levels by age. Continuous red lines: lower and upper reference limits; dashed gren lines: $90 \% \mathrm{Cls}$.

\section{Discussion}

In the present study, to obtain RI as effective as possible when applied to the study of women health, we selected from the general population women who were drug- and disease-free and having no signs or symptoms of androgen excess. Nonetheless, our cohort included women with unmedicated excess weight, dyslipidaemia, impaired insulin sensitivity and hypertension, overall affecting a relevant portion of the general population.

Our results confirmed the dramatic decline in circulating estrogens, progestins, androgens and precursors with menopause (28). No age dependency was detected for estrogen and progestin levels in reproductive age, therefore, the $60-90 \%$ hormone reduction observed after menopause could totally be attributed to ovarian senescence. At variance, the circulating androgens and precursors peaked around age 25 years and started a declining trend long before the menopausal transition. A steep decrease from age 25 to 65 years was observed for A4, DHEA 17OHP5, and DHT, ranging 40 to $85 \%$, while a moderate $20 \%$ decrease was found for $\mathrm{T}$, overall in good agreement with previous estimates $(29,30,31,32,33$, 


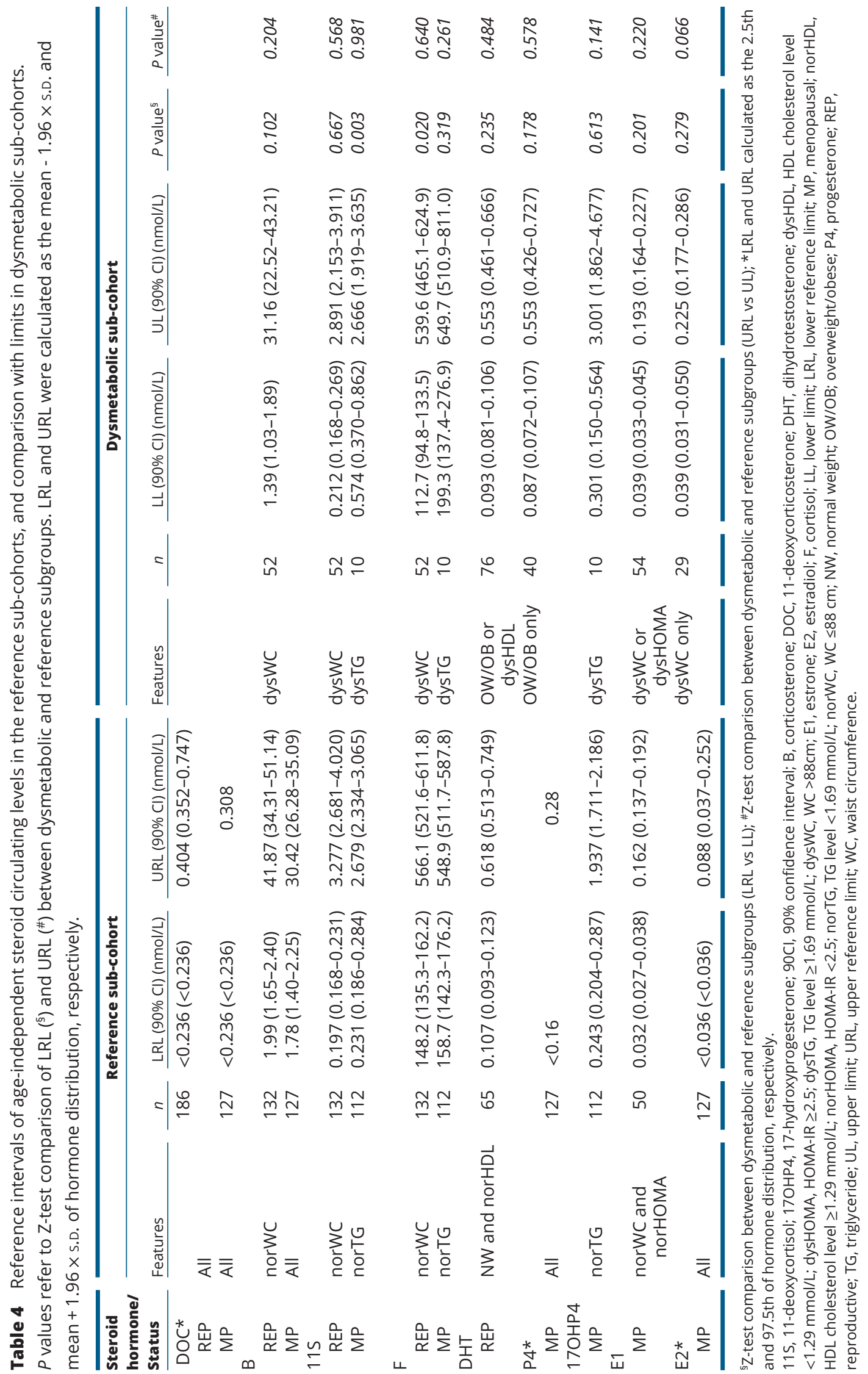


$34,35,36)$. Given the relevance of peripheral androgen generation from adrenal precursors in females, this finding may be due to the combined effect of ovarian and adrenal senescence (30). Interestingly, recent studies showed that zona reticularis, but not zona fasciculata, undergoes a large involution with aging, thus explaining the impairment of pro-androgen secretion $(33,36)$.

In our cohort, corticosteroid levels were not influenced by age, however, in keeping with a recent study (33), DOC, 11S and F slightly increased after menopause. This is in contrast with studies reporting an age-dependent reduction of glucocorticoids $(31,32)$. The modulation of steroid metabolites we observed suggests an increased adrenal secretion with menopause rather than a peripheral reactivation of $\mathrm{F}$ (37), as supported by an elegant study demonstrating a slight increase in menopausal $\mathrm{F}$ secretion in response to ACTH (30).

When we tried to disentangle which specific metabolic component related to a particular steroid level, we found a different network of relationships before and after menopause. Notably, in our non-hyperandrogenic women, no associations of androgens and precursors with metabolic parameters were found, except for DHT, inversely correlating with BMI and HDL-cholesterol in reproductive age. Unfortunately, the limited sensitivity of our assay prevented a similar DHT evaluation in menopausal women. The link between androgen levels and excess weight in women still has not been clarified. Indeed, a variegated combination of unchanged or reduced levels of DHEA, A4, T and DHT was associated with increasing adiposity in non-PCOS women by studies using high-specificity MS-based assays (31, 33, 34, 36, 38, $39,40,41)$. Interestingly, low plasma DHT was associated with increasing dimension and lipogenesis function of omental adipocytes (38). Our findings, therefore, support the concept that, in obese women, low levels of the most active androgen associate with the lipid storage capacity of visceral depots.

Interestingly, increasing estrogen levels contributed to the healthy lipid profile of our young women, underlying the importance of a balanced orchestration among active sex steroids. Besides, after menopause, E1 inversely associated with insulin resistance and directly associated with waist circumference. While, on the one hand, this is consistent with adipose tissue being the predominant estrogen source after ovarian senescence $(13,28)$, on the other hand, it contrasts with the detrimental link between abdominal obesity and insulin resistance. The beneficial involvement of estrogens in energy metabolism and inflammatory response was widely described $(28,42)$, 
and is evidenced by the vicious circle among abdominal adiposity, systemic low-grade inflammation, insulin resistance and increasing cardiovascular risk occurring with menopause (12). It is recognized that estrogen levels in menopause are proportional to body fat $(13,28)$; however, whether visceral or s.c. fat is the predominant source, and whether estrogens are associated with a favorable or unfavorable metabolic status in this life epoch, is still unclear. A study using LC-MS/MS found that visceral fat secreted $\mathrm{E} 1$ more than the s.c. fat in menopausal women, however, no correlations with the metabolic profile were performed (43). Another study found stronger associations of plasma estrogens with total rather than abdominal adiposity; moreover, direct associations between estrogens and insulin resistance were found, but these depended on the amount of fat (39). Given this scenario, our findings could be explained by the fact that, as we excluded medicated and diabetic subjects, our menopausal women are overall only mildly dysmetabolic. Unfortunately, in our population study we could not use techniques such as CT to distinguish between abdominal visceral and s.c. depots. Nonetheless, it is possible that fat amount reflected by waist circumference, be it s.c. or visceral, is prevalently metabolically healthy, so that the quantitative relationship between circulating E1, spillingover from fat depots, and the beneficial result of its function in terms of insulin sensitivity, is still detectable. Unfortunately, due to the limited analytical sensitivity, we could not investigate the metabolic associations of E2 in menopausal age.

Waist circumference was the only independent factor negatively affecting glucocorticoid levels in reproductive age. Moreover, menstrual phase-adjusted 17OHP4 levels, possibly reflecting its role as adrenal glucocorticoid precursor, diminished with increasing BMI, as reported in a recent study (31). This may appear in contrast with the direct link expected between glucocorticoid tone and visceral obesity. Nonetheless, the dynamic of HPA axis in obesity is complex, and early morning F levels were previously found unchanged or inversely related with central adiposity, as in consequence of diminished ACTH-sensitivity of the adrenal, of flattening of HPA circadian rhythmicity, or of increased F clearance $(44,45)$. At variance, the positive association of glucocorticoids with triglycerides we found in menopausal women is in line with their role in the derangement of energy substrate utilization (46). Notably, we demonstrated that even mild metabolic dysfunctions, such as high waist circumference or triglycerides, can significantly alter the estimation of $11 \mathrm{~S}$ and $\mathrm{F}$ limits, underlying the importance of a proper metabolic characterization when generating glucocorticoid RI.

Menstrual fluctuation challenges the definition of robust RI for ovarian steroids . A limit of our study is that gonadotropins were not measured, and menstrual classification was performed according to the menstrual date, which may represent a source of bias. We could partially cope with this unpredictable bias in the midluteal phase, by excluding women showing P4 levels $<10 \mathrm{nmol} / \mathrm{L}$ (27). However, we could not assess whether these women were actually misclassified or they had an occasional anovulatory cycle. Similarly, we cannot exclude that misclassification or anovulatory cycles were affecting women in early follicular and preovulatory phases. Therefore, our menstrual phase RI are to be interpreted with cautions, and need to be refined in future studies including gonadotropin evaluation. Nonetheless, values we observed in early follicular phase are in reasonable agreement with previous reports (27, $31,47)$. Androgen fluctuation throughout the menstrual cycle is still debated. Skiba et al. found slightly higher $\mathrm{T}$ and $\mathrm{A} 4$ in mid-cycle and luteal compared to follicular phase (34). Bui et al. observed minimal T fluctuation, with increasing values at mid-cycle in some women (48). We did not detect any androgen fluctuation, however, we found small but significant variations in DOC and 11S levels among menstrual phases. A proper exploration of this phenomenon would require repeated intra-subject evaluation across the menstrual cycle. Even though data are still inconclusive, we recommend to standardize the menstrual phase when studying the steroid dynamics in young women for clinical or research purposes.

Our steroid values are generally lower compared to other LC-MS/MS studies, except 17OHP5 and DHEA values which are higher, $(27,31,32,34,35,47,48,49,50)$. Differences in analytical methods, sampling procedures, cohort selection and study design may account for variabilities in steroid levels and relationships with women's metabolic health. Time of sampling (50) as well as needle stress $(51,52)$ represents non-negligible sources of variability. In addition, though harmonization of LC-MS/MS measures seems an affordable goal, collective strategies are still awaited in this direction.

In conclusion, our study focusing on a carefully selected female cohort highlighted a different network of relationships between circulating steroid profile, obesity and metabolic status of women in reproductive and menopausal age. RI specific for age, reproductive and menstrual status were generated that will be useful for 
the effective interpretation of the steroid involvement in women's health and disease.

\section{Supplementary materials}

This is linked to the online version of the paper at https://doi.org/10.1530/ EJE-20-1147.

\section{Declaration of interest}

The authors report no conflict of interest in this work. Alessandra Gambineri is on the editorial board of EJE. Alessandra Gambineri was not involved in the review or editorial process for this paper, on which he/she is listed as an author.

\section{Funding}

The study was supported by the European Union (REPROBESITY, FPVII-223,713), by the Emilia-Romagna Region - University programme 2007-2009 (g.a. PRUa1a-2007-006), by the Emilia-Romagna Region, Alessandro Liberati Young Researcher Grants (g.a. PRUA 1-2012-004).

\section{Author contribution statement}

M M 1 measured study samples, performed the statistical analysis and wrote the manuscript. A G, G D D, M B, V V and C P performed cohort recruitment and examination. A F and M M 2 measured study samples. U P designed the population study and wrote the manuscript. F F designed the study and wrote the manuscript.

\section{Acknowledgements}

The authors thank Shimadzu for kindly providing the LC-MS/MS platform used to generate part of the data used in this study.

\section{References}

1 Ketha SS, Singh RJ \& Ketha H. Role of mass spectrometry in clinical endocrinology. Endocrinology and Metabolism Clinics of North America 201746 593-613. (https://doi.org/10.1016/j. ecl.2017.04.001)

2 Wudy SA, Schuler G, Sánchez-Guijo A \& Hartmann MF. The art of measuring steroids: principles and practice of current hormonal steroid analysis. Journal of Steroid Biochemistry and Molecular Biology 2018179 88-103. (https://doi.org/10.1016/j.jsbmb.2017.09.003)

3 Ceriotti F, Hinzmann R \& Panteghini M. Reference intervals: the way forward. Annals of Clinical Biochemistry 200946 8-17. (https://doi. org/10.1258/acb.2008.008170)

4 Fanelli F \& Di Dalmazi G. Serum steroid profiling by mass spectrometry in adrenocortical tumors: diagnostic implications. Current Opinion in Endocrinology, Diabetes, and Obesity 201926 160-165. (https://doi.org/10.1097/MED.0000000000000475)

5 Gunness A, Pazderska A, Ahmed M, McGowan A, Phelan N, Boran G, Taylor AE, O'Reilly MW, Arlt W, Moore K, et al. Measurement of selected androgens using liquid chromatography-tandem mass spectrometry in reproductive-age women with Type 1 diabetes. Human Reproduction 201833 1727-1734. (https://doi.org/10.1093/ humrep/dey243)

6 Pasquali R, Zanotti L, Fanelli F, Mezzullo M, Fazzini A, MorselliLabate AM, Repaci A, Ribichini D \& Gambineri A. Defining hyperandrogenism in women with polycystic ovary syndrome: a challenging perspective. Journal of Clinical Endocrinology \& Metabolism 2016101 2013-2022. (https://doi.org/10.1210/jc.2015-4009)
7 O'Reilly MW, Kempegowda P, Jenkinson C, Taylor AE, Quanson JL, Storbeck KH \& Arlt W. 11-Oxygenated C19 steroids are the predominant androgens in polycystic ovary syndrome. Journal of Clinical Endocrinology \& Metabolism 2017102 840-848. (https://doi. org/10.1210/jc.2016-3285)

8 Sharma A, Kapoor E, Singh RJ, Chang AY \& Erickson D. Diagnostic thresholds for androgen-producing tumors or pathologic hyperandrogenism in women by use of total testosterone concentrations measured by liquid chromatography-tandem mass spectrometry. Clinical Chemistry 201864 1636-1645. (https://doi. org/10.1373/clinchem.2018.290825)

9 Turcu AF, El-Maouche D, Zhao L, Nanba AT, Gaynor A, Veeraraghavan P, Auchus RJ \& Merke DP. Androgen excess and diagnostic steroid biomarkers for nonclassic 21-hydroxylase deficiency without cosyntropin stimulation. European Journal of Endocrinology 2020183 63-71. (https://doi.org/10.1530/EJE-200129)

10 Fanelli F, Baronio F, Ortolano R, Mezzullo M, Cassio A, Pagotto U $\&$ Balsamo A. Normative basal values of hormones and protein of gonadal and adrenal functions from birth to adulthood. Sexual Development: Genetics, Molecular Biology, Evolution, Endocrinology, Embryology, and Pathology of Sex Determination and Differentiation 2018 12 50-94. (https://doi.org/10.1159/000486840)

11 Piché ME, Poirier P, Lemieux I \& Després JP. Overview of epidemiology and contribution of obesity and body fat distribution to cardiovascular disease: an update. Progress in Cardiovascular Diseases 201861 103-113. (https://doi.org/10.1016/j. pcad.2018.06.004)

12 Gerdts E \& Regitz-Zagrosek V. Sex differences in cardiometabolic disorders. Nature Medicine 201925 1657-1666. (https://doi. org/10.1038/s41591-019-0643-8)

13 Guarner-Lans V, Rubio-Ruiz ME, Pérez-Torres I \& Baños de MacCarthy G. Relation of aging and sex hormones to metabolic syndrome and cardiovascular disease. Experimental Gerontology 2011 46 517-523. (https://doi.org/10.1016/j.exger.2011.02.007)

14 Pasquali R \& Gambineri A. Polycystic ovary syndrome: a multifaceted disease from adolescence to adult age. Annals of the New York Academy of Sciences 20061092 158-174. (https://doi.org/10.1196/ annals.1365.014)

15 Pasquali R, Vicennati V, Gambineri A \& Pagotto U. Sex-dependent role of glucocorticoids and androgens in the pathophysiology of human obesity. International Journal of Obesity 200832 1764-1779. (https://doi.org/10.1038/ijo.2008.129)

16 Nieuwenhuizen AG \& Rutters F. The hypothalamic-pituitary-adrenal axis in the regulation of energy balance. Physiology and Behavior 2009 4 169-177.

17 Mezzullo M, Di Dalmazi G, Fazzini A, Baccini M, Repaci A, Gambineri A, Vicennati V, Pelusi C, Pagotto U \& Fanelli F. Impact of age, body weight and metabolic risk factors on steroid reference intervals in men. European Journal of Endocrinology 2020182 459-471. (https://doi.org/10.1530/EJE-19-0928)

18 Fanelli F, Belluomo I, Di Lallo VD, Cuomo G, De Iasio R, Baccini M, Casadio E, Casetta B, Vicennati V, Gambineri A, et al. Serum steroid profiling by isotopic dilution-liquid chromatography-mass spectrometry: comparison with current immunoassays and reference intervals in healthy adults. Steroids 201176 244-253. (https://doi. org/10.1016/j.steroids.2010.11.005)

19 Matthews DR, Hosker JP, Rudenski AS, Naylor BA, Treacher DF \& Turner RC. Homeostasis model assessment: insulin resistance and beta-cell function from fasting plasma glucose and insulin concentrations in man. Diabetologia 198528 412-419. (https://doi. org/10.1007/BF00280883)

20 Büttler RM, Martens F, Fanelli F, Pham HT, Kushnir MM, Janssen MJ, Owen L, Taylor AE, Soeborg T, Blankenstein MA, et al. Comparison of published LC-MS/MS methods for the simultaneous measurement of testosterone, androstenedione, and dehydroepiandrosterone 
in serum. Clinical Chemistry 201561 1475-1483. (https://doi. org/10.1373/clinchem.2015.242859)

21 Mezzullo M, Pelusi C, Fazzini A, Repaci A, Di Dalmazi G, Gambineri A, Pagotto U \& Fanelli F. Serum reference intervals for challenging sex and precursor steroids by liquid chromatography tandem mass spectrometry. Journal of Steroid Biochemistry and Molecular Biology 2020197105538.

22 Box GEP \& Cox DR. An analysis of transformations. Journal of the Royal Statistical Society: B 196426 211-243. (https://doi. org/10.1111/j.2517-6161.1964.tb00553.x)

23 Tukey JW Exploratory Data Analysis. Addison-Wesley, 1977. ISBN 9780-201-07616-5. OCLC 3058187.

24 Cohen J Statistical Power Analysis for the Behavioural Sciences. Routledge, 1988.

25 Clinical and Laboratory Standard Institute Defining, Establishing, and Verifying Reference Intervals in the Clinical Laboratory; Approved Guideline, 3rd ed. CLSI document EP28-A3c: Wayne, PA: Clinical and Laboratory Standards Institute, 2008.

26 Royston P \& Wright EM. Method for estimating age-specific reference intervals (normal ranges) based on fractional polynomials and exponential transformation. Journal of the Royal Statistical Society: Series A 1998161 79-101. (https://doi.org/10.1111/1467985X.00091)

27 Frederiksen H, Johannsen TH, Andersen SE, Albrethsen J, Landersoe SK, Petersen JH, Andersen AN, Vestergaard ET, Schorring ME, Linneberg A, et al. Sex-specific estrogen levels and reference intervals from infancy to late adulthood determined by LC-MS/MS. Journal of Clinical Endocrinology \& Metabolism 2020105 754-768. (https://doi.org/10.1210/clinem/dgz196)

28 Freeman EW, Sammel MD, Lin H \& Gracia CR. Obesity and reproductive hormone levels in the transition to menopause. Menopause 201017 718-726. (https://doi.org/10.1097/ gme.0b013e3181cec85d)

29 Kroboth PD, Salek FS, Pittenger AL, Fabian TJ \& Frye RF. DHEA and DHEA-S: a review. Journal of Clinical Pharmacology 199939 327-348. (https://doi.org/10.1177/00912709922007903)

30 Parker CR Jr, Slayden SM, Azziz R, Crabbe SL, Hines GA, Boots LR $\&$ Bae S. Effects of aging on adrenal function in the human: responsiveness and sensitivity of adrenal androgens and cortisol to adrenocorticotropin in premenopausal and postmenopausal women. Journal of Clinical Endocrinology \& Metabolism 200085 48-54. (https:// doi.org/10.1210/jcem.85.1.6265)

31 Eisenhofer G, Peitzsch M, Kaden D, Langton K, Pamporaki C, Masjkur J, Tsatsaronis G, Mangelis A, Williams TA, Reincke M, et al. Reference intervals for plasma concentrations of adrenal steroids measured by LC-MS/MS: impact of gender, age, oral contraceptives, body mass index and blood pressure status. Clinica Chimica Acta: International Journal of Clinical Chemistry 2017470 115-124. (https:// doi.org/10.1016/j.cca.2017.05.002)

32 Bae YJ, Zeidler R, Baber R, Vogel M, Wirkner K, Loeffler M, Ceglarek U, Kiess W, Körner A, Thiery J, et al. Reference intervals of nine steroid hormones over the life-span analyzed by LC-MS/MS: effect of age, gender, puberty, and oral contraceptives. Journal of Steroid Biochemistry and Molecular Biology 2019193 105409. (https:// doi.org/10.1016/j.jsbmb.2019.105409)

33 Nanba AT, Rege J, Ren J, Auchus RJ, Rainey WE \& Turcu AF. 11-Oxygenated C19 steroids do not decline with age in women. Journal of Clinical Endocrinology \& Metabolism $20191042615-2622$. (https://doi.org/10.1210/jc.2018-02527)

34 Skiba MA, Bell RJ, Islam RM, Handelsman DJ, Desai R \& Davis SR. Androgens During the reproductive years: what is normal for women? Journal of Clinical Endocrinology \& Metabolism 2019104 5382-5392. (https://doi.org/10.1210/jc.2019-01357)

35 van der Veen A, van Faassen M, de Jong WHA, van Beek AP, DijckBrouwer DAJ \& Kema IP. Development and validation of a LC-MS/MS method for the establishment of reference intervals and biological variation for five plasma steroid hormones. Clinical Biochemistry 2019 68 15-23. (https://doi.org/10.1016/j.clinbiochem.2019.03.013)

36 Davio A, Woolcock H, Nanba AT, Rege J, O'Day P, Ren J,

Zhao L, Ebina H, Auchus R, Rainey WE, et al. Sex differences in 11-oxygenated androgen patterns Across adulthood. Journal of Clinical Endocrinology \& Metabolism 2020105 1-9. (https://doi. org/10.1210/clinem/dgaa343)

37 Yamatani H, Takahashi K, Yoshida T, Takata K \& Kurachi H. Association of estrogen with glucocorticoid levels in visceral fat in postmenopausal women. Menopause 201320 437-442. (https://doi. org/10.1097/gme.0b013e318271a640)

38 Côté JA, Lessard J, Mailloux J, Laberge P, Rhéaume C \& Tchernof A. Circulating $5 \alpha$-dihydrotestosterone, abdominal obesity and adipocyte characteristics in women. Hormone Molecular Biology and Clinical Investigation 201212 391-400. (https://doi.org/10.1515/hmbci-20120026)

39 Marchand GB, Carreau AM, Weisnagel SJ, Bergeron J, Labrie F, Lemieux $S \&$ Tchernof A. Increased body fat mass explains the positive association between circulating estradiol and insulin resistance in postmenopausal women. American Journal of Physiology. Endocrinology and Metabolism 2018314 E448-E456. (https://doi. org/10.1152/ajpendo.00293.2017)

40 Marchand GB, Carreau A, Laforest S, Côté J, Daris M, Cianflone K, Prehn C, Adamski J \& Tchernof A. Circulating steroid levels as correlates of adipose tissue phenotype in premenopausal women. Hormone Molecular Biology and Clinical Investigation 201834 20170082 (https://doi.org/10.1515/hmbci-2017-0082).

41 Vihma V, Heinonen S, Naukkarinen J, Kaprio J, Rissanen A, Turpeinen U, Hämäläinen E, Hakkarainen A, Lundbom J, Lundbom $\mathrm{N}$, et al. Increased body fat mass and androen metabolism—a twin study in healthy young women. Steroids 2018 140 24-31. (https://doi.org/10.1016/j.steroids.2018.08.006)

42 Faulds MH, Zhao C, Dahlman-Wright K \& Gustafsson JÅ. The diversity of sex steroid action: regulation of metabolism by estrogen signaling. Journal of Endocrinology 2012212 3-12. (https://doi. org/10.1530/JOE-11-0044)

43 Hetemäki N, Savolainen-Peltonen H, Tikkanen MJ, Wang F, Paatela H, Hämäläinen E, Turpeinen U, Haanpää M, Vihma V \& Mikkola TS. Estrogen metabolism in abdominal subcutaneous and visceral adipose tissue in postmenopausal women. Journal of Clinical Endocrinology \& Metabolism 2017102 4588-4595. (https://doi. org/10.1210/jc.2017-01474)

44 Walker BR, Soderberg S, Lindahl B \& Olsson T. Independent effects of obesity and cortisol in predicting cardiovascular risk factors in men and women. Journal of Internal Medicine 2000247 198-204. (https:// doi.org/10.1046/j.1365-2796.2000.00609.x)

45 Roelfsema F, Pereira AM \& Veldhuis JD. Impact of adiposity and fat distribution on the dynamics of adrenocorticotropin and cortisol rhythms. Current Obesity Reports 20143 387-395. (https://doi. org/10.1007/s13679-014-0118-7)

46 Björntorp P \& Rosmond R. Obesity and cortisol. Nutrition 200016 924-936. (https://doi.org/10.1016/s0899-9007(00)00422-6)

47 Verdonk SJE, Vesper HW, Martens F, Sluss PM, Hillebrand JJ \& Heijboer AC. Estradiol reference intervals in women during the menstrual cycle, postmenopausal women and men using an LC-MS/MS method. Clinica Chimica Acta: International Journal of Clinical Chemistry 2019495 198-204. (https://doi.org/10.1016/j. cca.2019.04.062)

48 Bui HN, Sluss PM, Blincko S, Knol DL, Blankenstein MA \& Heijboer AC. Dynamics of serum testosterone during the menstrual cycle evaluated by daily measurements with an ID-LC-MS/MS method and a 2nd generation automated immunoassay. Steroids 2013 78 96-101. (https://doi.org/10.1016/j.steroids.2012.10.010)

49 Kushnir MM, Rockwood AL, Roberts WL, Pattison EG, Owen WE, Bunker AM \& Meikle AW. Development and performance evaluation of a tandem mass spectrometry assay for 4 adrenal steroids. 
Clinical Chemistry 200652 1559-1567. (https://doi.org/10.1373/ clinchem.2006.068445)

50 Parikh TP, Stolze B, Ozarda Y, Jonklaas J, Welsh K, Masika L, Hill M, DeCherney A \& Soldin SJ. Diurnal variation of steroid hormones and their reference intervals using mass spectrometric analysis. Endocrine Connections 20187 1354-1361. (https://doi.org/10.1530/ EC-18-0417)

51 Weckesser LJ, Plessow F, Pilhatsch M, Muehlhan M, Kirschbaum C $\&$ Miller R. Do venepuncture procedures induce cortisol responses? A review, study, and synthesis for stress research, study. Psychoneuroendocrinology 201446 88-99. (https://doi.org/10.1016/j. psyneuen.2014.04.012)

52 Mezzullo M, Fanelli F, Di Dalmazi G, Fazzini A, Ibarra-Gasparini D, Mastroroberto M, Guidi J, Morselli-Labate AM, Pasquali R, Pagotto U, et al. Salivary cortisol and cortisone responses to short-term psychological stress challenge in late adolescent and young women with different hyperandrogenic states. Psychoneuroendocrinology 2018 91 31-40. (https://doi.org/10.1016/j.psyneuen.2018.02.022)

Received 5 October 2020

Revised version received 10 December 2020

Accepted 12 January 2021 